\title{
Perfil epidemiológico de las fracturas mandibulares en el servicio de urgencias
}

\author{
Epidemiological profile of mandibular fractures in an emergency department
}

\begin{abstract}
Beatriz Villavicencio-Ayala1, David Rojano-Mejía², Jorge Quiroz-Williams² y Álvaro Albarrán-Becerri2*
${ }^{1}$ Servicio de Cirugía Maxilofacial, Hospital General Ticomán, Secretaría de Salud del Distrito Federal; ${ }^{2}$ Unidad Médica de Alta Especialidad de Traumatología, Ortopedia y Rehabilitación Dr. Victorio de la Fuente Narváez Hospital de Traumatología, Instituto Mexicano del Seguro Social. Ciudad de México, México
\end{abstract}

\section{Resumen}

Objetivo: Identificar el perfil epidemiológico de las fracturas mandibulares en el servicio de urgencias. Método: Estudio observacional, retrospectivo, en el que se incluyeron expedientes de derechohabientes con diagnóstico de fractura mandibular tratados entre el 1 de enero de 2015 y el 30 de junio de 2019 en el servicio de urgencias del Hospital de Traumatología Dr. Victorio de la Fuente Narváez. De los expedientes se obtuvieron la edad, el sexo, la ocupación, la comorbilidad, el antecedente de toxicomanías y la prevalencia de las fracturas mandibulares de acuerdo con el número de fracturas y la zona anatómica. Resultados: Se incluyeron 319 sujetos con fracturas mandibulares, jóvenes (32.5 años), con predominio del sexo masculino (89.7\%), la mayoría desarrollaba algún oficio (64.2\%), el 14.4\% con alguna comorbilidad y el 76\% presentaba alcoholismo al momento de la lesión. El sitio anatómico más afectado fue el ángulo mandibular, seguido de la parasínfisis. La causa más frecuente de lesión fue por agresión física. Conclusiones: Las fracturas mandibulares en nuestro servicio de urgencias suelen ser simples y afectar el ángulo de la mandíbula, presentándose principalmente en hombres en la tercera década de la vida. Las agresiones son el mecanismo de lesión mas frecuente y por lo general se asocian al consumo de sustancias tóxicas.

Palabras clave: Fracturas mandibulares. Prevalencia. Agresiones físicas. Factor de riesgo.

\section{Abstract}

Objective: To identify the epidemiological profile of mandibular fractures in the emergency department. Method: Observational, retrospective study included records of persons with a diagnosis of mandibular fracture treated between January 1, 2015 and June 30, 2019, from the emergency department of the Hospital de Traumatología Dr. Victorio de la Fuente Narváez. The age, sex, occupation, comorbidities, prevalence of mandibular fractures according to the number of fractures and anatomical area, were obtained from the clinical records. Results: 319 subjects with mandibular fractures were included in the study, which were young people (32.5 years) of the male sex (89.7\%), the majority developed some trade (64.2\%), $14.4 \%$ had some comorbidity, $76 \%$ had alcoholism when time of injury. The most affected anatomical site was the mandibular angle followed by the parasymphysis, the most frequent cause of injury was due to physical aggression. Conclusions: Mandibular fractures in our emergency department are usually simple, mainly affecting the angle of the jaw, occurring mainly in men in their third decade of life, assaults are the most frequent injury mechanism and are generally associated with substance use toxic.

Key words: Mandibular fractures. Prevalence. Physical aggressions. Risk factor.

\section{Correspondencia:}

*Álvaro Albarrán-Becerril

Avda. Fortuna s/n

Magdalena de las Salinas, Gustavo A. Madero

Fecha de recepción: 09-08-2020

C.P. 07760 , Ciudad de México, México

Fecha de aceptación: 03-02-2021

E-mail: rojanodavid@gmail.com

DOI: $10.24875 / C I R U .200008811$

Cir Cir. 2021;89(5):646-650

Contents available at PubMed www.cirugiaycirujanos.com 0009-7411/@ 2021 Academia Mexicana de Cirugía. Publicado por Permanyer. Este es un artículo open access bajo la licencia CC BY-NC-ND (http://creativecommons.org/licenses/by-nc-nd/4.0/). 


\section{Introducción}

Las fracturas mandibulares ocupan el segundo lugar entre las fracturas faciales (superadas únicamente por las fracturas de los huesos nasales) y el décimo entre las fracturas de todo el organismo. Se calcula que anualmente las fracturas mandibulares suponen el $36 \%$ de todas las fracturas del complejo maxilofacial. Estas fracturas requieren un tratamiento inmediato de urgencia y un personal altamente cualificado, ya que en ocasiones puede aparecer un grave compromiso respiratorio que comprometa la vida del paciente. En los últimos años, estas fracturas han tenido un incremento en su prevalencia debido al mayor número de lesiones ocasionadas por vehículos automotores $^{1,2}$. En todo el mundo, según diversos estudios, los accidentes de tránsito son la principal causa de fracturas, tanto de la cara como de otras partes del cuerpo ${ }^{3-7}$, seguidos de los accidentes de moto, los atropellos por vehículos y los accidentes en bicicleta, siendo las carreteras el lugar donde más se reportaron estos accidentes ${ }^{8,9}$. Sin embargo, en México, a diferencia de lo reportado en la literatura, el principal agente etiológico para las fracturas mandibulares es la violencia interpersonal ${ }^{10}$.

Hay varias clasificaciones de las fracturas mandibulares de acuerdo con diversos criterios, como la relación entre los fragmentos de la fractura ${ }^{11}$, la presencia o no de dientes en los trazos de fractura ${ }^{12}$, o la inserción muscular, siendo una de las clasificaciones más utilizadas la de Dingman y Natvig, que considera la localización anatómica de la fractura ${ }^{13}$.

Algunos autores mencionan que la zona parasinfisaria es de las más afectadas en las fracturas mandibulares ${ }^{14,15}$; sin embargo, otros reportan que es el cuerpo mandibular ${ }^{16}$. En cuanto a la edad de presentación de las fracturas mandibulares, la mayoría ocurren entre los 16 y los 30 años $^{17}$. Dentro de los factores de riesgo para presentar fracturas mandibulares se encuentra el antecedente de toxicomanías (alcohol y drogas) y vivir en ciudades donde hay una alta tasa de desempleo y de consumo de alcoholis,19.

En México no hay reportes recientes del perfil epidemiológico de las fracturas mandibulares en el servicio de urgencias, por lo que identificar el perfil epidemiológico de dichas fracturas permitirá establecer estrategias para mejorar la calidad en la atención médica de estos pacientes.

El objetivo principal del estudio es determinar la edad, el sexo, la ocupación, la comorbilidad, el antecedente de toxicomanías y la prevalencia de las fracturas mandibulares de acuerdo con el número de fracturas y la zona anatómica, en el servico de urgencias del Hospital de Traumatología Dr. Victorio de la Fuente Narváez.

\section{Método}

Se realizó un estudio observacional, retrospectivo, analítico, de 319 pacientes con diagnóstico de fractura mandibular tratados en el departamento clínico de cirugía maxilofacial del Hospital de Traumatología, perteneciente a la Unidad Médica de Alta Especialidad de Traumatología, Ortopedia y Rehabilitación Dr. Victorio de la Fuente Narváez, del Instituto Mexicano del Seguro Social, del 1 de enero de 2015 al 30 de junio de 2019. Se incluyeron pacientes derechohabientes sin distinción de sexo, con edades comprendidas de 17 a 65 años, con o sin comorbilidad, con expediente clínico físico y electrónico completo. Se excluyeron los pacientes que no contaban con expediente clínico completo, aquellos con falta de seguimiento en consulta externa y los que presentaran fractura mandibular por otras causas no traumáticas. Se recabaron las siguientes variables: edad, sexo, ocupación (oficio, trabajador administrativo, estudiante, ayudante general, profesionista, desempleado, jubilado o pensionado, obrero, ama de casa y trabajador de la construcción), comorbilidad (diabetes mellitus, hipertensión arterial sistémica), toxicomanía previa o al momento de la lesión (alcoholismo, tabaquismo u otro tipo de drogas, como anfetaminas, inhalantes, marihuana, cocaína y otras), número de fracturas y zona anatómica (sínfisis, parasínfisis, cuerpo, ángulo, rama mandibular o apófisis coronoides).

Las variables cualitativas se presentaron como frecuencias absolutas y relativas, y las variables cuantitativas como medidas de tendencia central (media) y de dispersión (desviación estándar).

El estudio fue aprobado por el Comité Local de Investigación y el Comité Local de Ética en Investigación de Salud de nuestro centro.

\section{Resultados}

En el estudio se incluyeron 319 pacientes con fracturas mandibulares, de los cuales 286 eran del sexo masculino $(89.7 \%)$, con una edad promedio de 32.5 años $( \pm 2.04)$. La ocupación más frecuente fue el desempeño de algún oficio (64.2\%) y el resto realizaba trabajo administrativo (35.7\%). Con relación a la 
comorbilidad, solo el $14.42 \%$ presentó alguna. La toxicomanía más frecuente al momento del accidente fue el alcoholismo (76.17\%), seguido del tabaquismo $(57.9 .2 \%)$ y del consumo de otro tipo de drogas $(21.9 \%)$.

En lo que respecta al número de fracturas, $159(49.8 \%)$ sujetos presentaron fracturas mandibulares simples o únicas, 152 (47.6\%) presentaron fracturas dobles y $8(2.5 \%)$ tuvieron fracturas triples. Con relación a las fracturas según la zona anatómica, más del $60 \%$ de los casos correspondieron a dos tipos de fracturas: las que involucraban el ángulo y las que involucraban la parasínfisis (Tabla 1).

En cuanto al mecanismo de la lesión, las fracturas de mandíbula fueron provocadas en su mayoría (213) por traumas directos (agresiones por asalto, por riña, por accidentes de trabajo y deportivos), seguidos por los accidentes de moto (Tabla 2).

\section{Discusión}

En este estudio se analizaron 319 pacientes con fracturas mandibulares entre la segunda y tercera década de la vida, siendo la edad promedio de 32.5 años, similar a la reportado en otros estudios ${ }^{19-21}$. Esto se debe a que los jóvenes tienen una mayor participación que otros grupos de edad en la sociedad en actividades al aire libre, y por lo tanto son más propensos a sufrir traumas en diversas actividades físicas, conducción imprudente, abuso de alcohol, violencia interpersonal y diferentes actividades deportivas ${ }^{22}$.

Las fracturas mandibulares se presentaron preferentemente en el sexo masculino (89.7\%), lo cual coincide con otros estudios ${ }^{18-21,23}$ en los que el número de hombres afectados fue aproximadamente ocho veces mayor que el de mujeres. La mayor frecuencia de fracturas en los hombres es apoyada por el hecho de que en este estudio la violencia es la causa principal de las fracturas mandibulares, como también mencionan Ellis, et al. ${ }^{7}$ en su estudio.

En cuanto a la ocupación, la mayoría de los pacientes con fractura mandibular desempeñaban oficios, probablemente debido al mayor desplazamiento que requiere el trayecto laboral en una ciudad con alto tráfico, además de realizar tareas que requieren mayor esfuerzo físico por el uso de herramientas pesadas ${ }^{24}$.

Con relación a las comorbilidad, solo la presentaban el $14.4 \%$ de los sujetos del estudio, ya que los pacientes en su mayoría eran adultos jóvenes, especialmente en la tercera década de la vida; lo anterior
Tabla 1. Tipos de fractura según la zona anatómica

\begin{tabular}{lll}
\hline Zona anatómica & N & $\%$ \\
\hline Ángulo & 85 & 26.6 \\
Parasínfisis y ángulo & 76 & 23.8 \\
Parasínfisis & 35 & 11 \\
Cuerpo & 24 & 7.5 \\
Parasínfisis y subcondílea & 14 & 4.4 \\
Cuerpo y ángulo & 14 & 4.4 \\
Parasínfisis y cóndilo & 8 & 2.5 \\
Triple & 8 & 2.5 \\
Subcondílea & 6 & 1.9 \\
Parasínfisis y rama & 5 & 1.6 \\
Cóndilo & 5 & 1.6 \\
Ángulo y subcondílea & 5 & 1.6 \\
Rama & 4 & 1.3 \\
Parasínfisis y cuerpo & 4 & 1.3 \\
Ángulo y cóndilo & 4 & 1.3 \\
Otras & 22 & 6.7 \\
\hline
\end{tabular}

Tabla 2. Mecanismo de la lesión

\begin{tabular}{lcc}
\hline Mecanismo & N & $\%$ \\
\hline Trauma directo & 213 & 66.8 \\
Accidente de motocicleta & 32 & 10 \\
Accidente de automóvil & 24 & 7.5 \\
Caída de $<2 \mathrm{~m}$ & 21 & 6.6 \\
Arma de fuego & 16 & 5 \\
Caída de $>2 \mathrm{~m}$ & 13 & 4.1 \\
\hline
\end{tabular}

es importante porque la comorbilidad contribuye a un riesgo incrementado de presentar complicaciones ${ }^{24}$.

Es este estudio, la fractura que más se presentó fue la de ángulo mandibular, seguida de la fractura doble de parasínfisis y ángulo, en concordancia con lo reportado por otros estudios ${ }^{17,19,21,25}$.

En cuanto al mecanismo de la lesión de las fracturas mandibulares, estas fueron provocadas en su mayoría por agresiones físicas (riña o asalto), rebasando en la actualidad a las fracturas por accidente automovilísticos, lo cual coincide con lo reportado en otras investigaciones ${ }^{7,14,17-19,24}$, quedando en segundo lugar 
los accidentes de moto y en tercero los accidentes automovilísticos. Lo anterior difiere de lo reportado en otros estudios ${ }^{3-6}$ debido probablemente a diferencias geográficas y culturales, estatus socioeconómico, cambios en el estilo de vida, el transporte y las medidas legislativas.

En cuanto a los accidentes de moto, el uso de casco se reportó solo en 11 de 32 pacientes al momento del accidente, lo cual influyó en la disminución del impacto traumático. Esto es importante, ya que el uso de un casco que cumpla con las normas vigentes, al igual que la utilización de cinturones de seguridad en los automóviles y de protectores faciales en deportes extremos o de alto riesgo y trabajos peligrosos, son medidas de gran importancia para disminuir la morbilidad por trauma maxilofacial ${ }^{26}$.

En cuanto a las toxicomanías previas o al momento de la lesión, nuestros resultados demuestran que esta condición es muy frecuente, lo cual es similar a lo reportado por Mathog, et al. ${ }^{27}$, debido probablemente a que el consumo de alcohol se considera parte del estilo de vida en las generaciones actuales y existe una evidencia clara de que el consumo agudo de alcohol aumenta el riesgo de padecer algún tipo de lesión o de verse implicado en situaciones violentas, caídas 0 accidentes de tráfico ${ }^{28}$. Igualmente, se ha publicado un aumento de la mortalidad y de la morbilidad en pacientes que presentan lesiones faciales y son consumidores de alcohol o drogas, puesto que la cara suele ser la región diana en la violencia relacionada con el consumo de tóxicos ${ }^{29}$. Por todo ello, en nuestro estudio recabamos los datos de consumo de tóxicos en la población estudiada, y observamos que la mayoría de los casos estaban relacionados principalmente con el alcohol.

La utilidad de estos resultados reside en que deben llevarse a cabo medidas preventivas específicas en la población con más frecuencia afectada, ya que esto es un problema de salud pública que va en aumento. También hay que concienciar a los pacientes y la sociedad con respecto al daño en la integridad física al que están expuestos al consumir alcohol y otras drogas, además de que el antecedente de toxicomanías aumenta la probabilidad de tener un accidente y, en caso de que ocurra, la complejidad de las fracturas se incrementa, requiriendo una atención oportuna y especializada por un equipo multidisciplinario y mayores tratamientos quirúrgicos que resultarán en una carga económica para las instituciones y un pronóstico menos favorable para el paciente.
Trabajos como este tienen como desventaja que se basan en expedientes clínicos, los cuales pudieron obviar otras lesiones relacionadas. También, en ocasiones, la información no está completa, y además los datos recolectados pertenecen al área norte de la Ciudad de México y al Estado de Hidalgo, cuyas características demográficas y sociales son diferentes de las de otros Estados, por lo que nuestros resultados no deben extrapolarse a toda la población mexicana.

Con este estudio buscamos aportar información estadística de las fracturas mandibulares que más comúnmente se presentan en el servicio de cirugía maxilofacial del Hospital de Traumatología Dr. Victorio de la Fuente Narváez; sin embargo, aún falta mucha información en nuestro país que haga referencia a la traumatología maxilofacial y más en específico a las fracturas mandibulares. Es de suma importancia conocer y documentar todos los casos que se atienden en las diferentes unidades hospitalarias del país para procesar la información y realizar publicaciones que nos ayuden a brindar un mejor tratamiento a nuestros pacientes.

\section{Conclusiones}

Las fracturas mandibulares en nuestro servicio de urgencias suelen ser simples y afectar principalmente el ángulo de la mandíbula, se presentan principalmente en hombres en la tercera década de la vida, las agresiones son el mecanismo de lesión más frecuente y por lo general se asocian al consumo de sustancias tóxicas.

\section{Responsabilidades éticas}

Protección de personas y animales. Los autores declaran que para esta investigación no se han realizado experimentos en seres humanos ni en animales.

Confidencialidad de los datos. Los autores declaran que han seguido los protocolos de su centro de trabajo sobre la publicación de datos de pacientes.

Derecho a la privacidad y consentimiento informado. Los autores declaran que en este artículo no aparecen datos de pacientes.

\section{Conflicto de intereses}

Los autores declaran no tener ningún conflicto de intereses. 


\section{Bibliografía}

1. Santiago MJG, Pérez SA, Suárez RAS, Barrios JLL. Incidencia de fracturas mandibulares. Revisión de 634 casos en 493 pacientes. Rev Mex Cirugía Bucal y Maxilofac. 2017;13:95-9.

2. Exadaktylos AK, Eggensperger NM, Eggli S, Smolka KM, Zimmermann H, lizuka T. Sports related maxillofacial injuries: the first maxillofacial trauma database in Switzerland. Br J Sports Med. 2004;38:750-3.

3. Tanaka N, Tomitsuka K, Shionoya K, Andou H, Kimijima Y, Tashiro T, et al. Aetiology of maxillofacial fracture. $\mathrm{Br} \mathrm{J}$ Oral Maxillofac Surg. 1994;32:19-23.

4. Ferreira PC, Amarante JM, Silva PN, Rodrigues JM, Choupina MP Silva AC, et al. Retrospective study of 1251 maxillofacial fractures in children and adolescents. Plast Reconstr Surg. 2005;115:1500-8.

5. Chrcanovic BR, Freire-Maia B, De Souza LN, De Araújo VO, De Abreu MHNG. Facial fractures: a 1-year retrospective study in a hospital in Belo Horizonte. Braz Oral Res. 2004;18:322-8.

6. Al Ahmed HE, Jaber MA, Abu Fanas SH, Karas M. The pattern of maxillofacial fractures in Sharjah, United Arab Emirates: a review of $230 \mathrm{ca}-$ ses. Oral Surg Oral Med Oral Pathol Oral Radiol Endod. 2004;98:166-70.

7. Ellis E, Moos KF, el-Attar A. Ten years of mandibular fractures: an analysis of 2,137 cases. Oral Surg Oral Med Oral Pathol. 1985;59:120-9.

8. Klenk G, Kovacs A. Etiology and patterns of facial fractures in the United Arab Emirates. J Craniofac Surg. 2003;14:78-84.

9. Aksoy $E$, Unlü $E$, Sensöz O. A retrospective study on epidemiology and treatment of maxillofacial fractures. J Craniofac Surg. 2002;13:772-5.

10. Gamboa R, Vargas G, Casafont A. Análisis de la frecuencia de fracturas faciales atendidas por el Servicio de Cirugía Maxilofacial del Hospital San Juan de Dios, entre 2007 y 2010. Rev Mex Cirugía Bucal y Maxilofac. 2013;9:4-9.

11. Portaceli T, Picón M, García A. Fracturas mandibulares. En: Protocolos clínicos de la Sociedad Española de Cirugía Oral y Maxilofacial. 3.ㄹ ed. Madrid, España: SCPRE; 2014. p. 183-92.

12. Kazanjian VH, Converse JM. Surgical treatment of facial injuries. $3^{\text {rd }}$ ed Baltimore: Williams \& Wilkins; 1975

13. Dingman RO. Surgery of facial fractures. Philadelphia, USA: W.B. Saunders; 1964. 245 p.

14. Afrooz PN, Bykowski MR, James IB, Daniali LN, Clavijo-Alvarez JA. The epidemiology of mandibular fractures in the United States, Part 1: A review of 13,142 cases from the US National Trauma Data Bank. J Oral Maxillofac Surg. 2015;73:2361-6.

15. Boffano P, Kommers SC, Karagozoglu KH, Forouzanfar T. Aetiology of maxillofacial fractures: a review of published studies during the last 30 years. Br J Oral Maxillofac Surg. 2014;52:901-6.
16. Dongas $P$, Hall GM. Mandibular fracture patterns in Tasmania, Australia. Aust Dent J. 2002;47:131-7.

17. Pacheco MA, Rodríguez MA. Fracturas mandibulares: estudio de 5 años en el Hospital Central Militar de México. An Otorrinolaringol Mex. 2007:52:150-3.

18. Castillo CD, Gabriela MM, Blanco S, Kauan M, Martínez RAG, Linares M, et al. Prevalencia de fracturas en los maxilares de los pacientes que acudieron al servicio de cirugía maxilo facial del hospital clínico universitario (HCU): febrero-noviembre 2004. Acta Odontol Venez. 2006;44:357-63.

19. Medina-Solis CE, Córdova-González JL, Casanova-Rosado AJ, Zazueta-Hernández MA. Fracturas maxilofaciales y factores asociados en derechohabientes del IMSS Campeche, México: Análisis retrospectivo 1994-1999. Gac Med Mex. 2004;140:27-32.

20. Shah N, Patel S, Sood R, Mansuri Y, Gamit M, Rupawala T. Analysis of mandibular fractures: a 7-year retrospective study. Ann Maxillofac Surg. 2019;9:349-54.

21. Zapata S, Pacheco C, Núñez C, Gazitúa G, Cerda P. Epidemiología de las fracturas mandibulares tratadas quirúrgicamente en el Instituto Traumatológico de Santiago (Chile): 10 años de revisión. Rev Esp Cir Oral Maxilofac. 2015;37:138-43.

22. De Pablo B, Ontañón AV, Font GR. Fracturas mandibulares: manejo en deportistas. Apunt Med l'Esport. 2017;52:123-7.

23. Quintana Díaz JC, Ali Zwiad A, Pinilla González R, López Lazo S, Maestre Márquez H, Quintana Giralt M. Comportamiento de las fracturas maxilofaciales atendidas en el Hospital Universitario de Maabar, República de Yemen. Rev Cuba Cirugía. 2012;51:1-9.

24. González Mora LE Vargas Farren I, Pedemonte Trewhela C Canales Trkovic M, Sáez Salas F, Verdugo-Avello F. Análisis de las fracturas mandibulares causadas por accidentes laborales: estudio descriptivo retrospectivo. Int J Odontostomatol. 2015:9:198-203.

25. Raposo A, Preisler G, Salinas F, Muñoz C, Monsalves MJ. Epidemiología de las fracturas maxilofaciales tratadas quirúrgicamente en Valdivia, Chile: 5 años de revisión. Rev Esp Cir Oral Maxilofac. 2013;35:18-22.

26. Ortiz G, Arango JC, Giraldo C, Ramírez D, Uribe JC. Análisis retrospectivo de historias clínicas de pacientes intervenidos por cirugía maxilofacial en el Hospital General de Medellín. Ces Odontol. 2007;20:17-21.

27. Mathog $\mathrm{RH}$, Toma V, Clayman L, Wolf $\mathrm{S}$. Nonunion of the mandible: an analysis of contributing factors. J Oral Maxillofac Surg. 2000;58:746-52.

28. Porpiglia NM, Bortolotti F, Dorizzi RM, Micciolo R, Tagliaro F. Critical evaluation of the association between elevated mean corpuscular volume and alcohol-related traffic accidents: a retrospective study on 6244 car crash cases. Alcohol Clin Exp Res. 2019;43:1528-32.

29. Valen A, Bogstrand ST, Vindenes V, Frost J, Larsson M, Holtan A, et al. Driver-related risk factors of fatal road traffic crashes associated with alcohol or drug impairment. Accid Anal Prev. 2019;131:191-9. 